\title{
Masson's tumor and calcifying aponeurotic fibroma: two rare soft- tissue lesions in the same finger. High-resolution ultrasound features with histopathological correlations
}

\author{
Nicola Romano ${ }^{1}$, Aldo Fischetti ${ }^{1}$, Ilaria Mussettoํ, Rita Bianchi², Alessandro Muda ${ }^{3}$
}

${ }^{1}$ Department of Radiology, University of Genoa, ${ }^{2}$ Pathology Unit, University of Genoa ${ }^{3}$ Department of Radiology, Ospedale Policlinico San Martino, Genoa, Italy

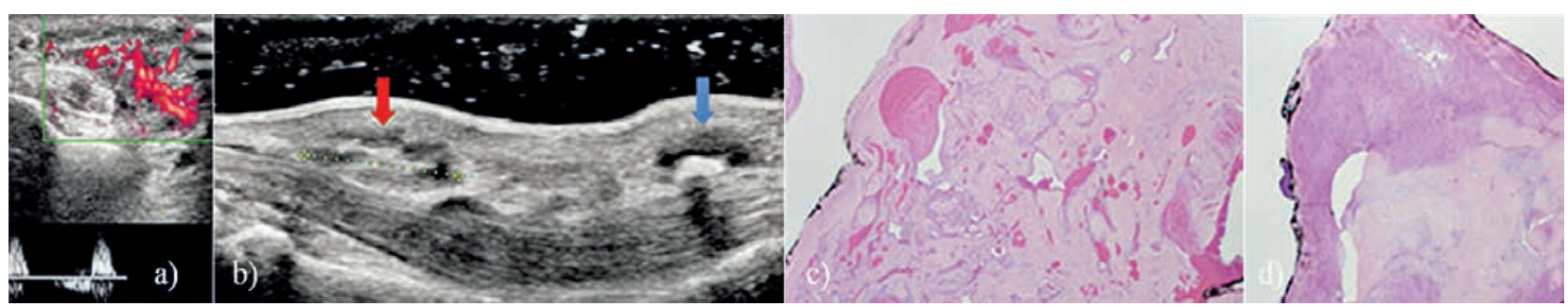

Fig 1. HRUS (16MHz), longitudinal view: a) greater lesion with increased vascularization (systolic flow) at power Doppler examination: nodular inhomogeneous solid formation of $1,7 \mathrm{~cm}$ and cleaved from the flexor tendons (red arrow); b) the smaller lesion (blue arrow) with a calcification was in close relationship with flexor tendons; c) Masson's tumor (Haematoxylin-Eosin-20x); d) Calcifying aponeurotic fibroma (Haematoxylin-Eosin-20x)

\section{To the editor,}

A 71-year old man presented with two soft tissue masses on the $3^{\text {rd }}$ finger of left hand. The largest, with soft consistency, was localized at the level of the palmar aspect of the proximal phalanx, and the smaller one, with hard consistency, was localized at metacarpophalangeal joint level. High resolution ultrasound (HRUS) examination showed the presence of a $1.7 \mathrm{~cm}$ nodular inhomogeneous solid mass, cleaved from the flexor tendons with an increased vascularization (systolic flow) on power Doppler examination (fig 1a). The smaller lesion measured $1 \mathrm{~cm}$ and was partially vascularized with a calcification, and was in close relationship to the flexor tendons, that were sliding normally on dynamic maneuvers (fig 1b). One month later the

Received 29.08.2017 Accepted 29.09.2017

Med Ultrason

2017, Vol. 19, No 4, 457-458, DOI: 10.11152/mu-1251

Corresponding author: Nicola Romano, MD, Department of Radiology, University of Genoa, Italy

Via Leon Battista Alberti 4, 16132, Genoa, Italy

Phone: +393356321624

E-mail: romano.nicola@live.it surgical excision was performed. The post-operative histopathology examination confirmed the diagnosis of Masson's tumor for the greater mass (fig 1c) and of calcifying aponeurotic fibroma for the smaller lesion (fig 1d). No previous case with this association has been reported in literature.

Masson's Tumor (or intravascular papillary endothelial hyperplasia- IPEH) is a rare vascular lesion ( $2-4 \%$ of the vascular tumors of the skin and soft tissues) characterized by proliferation of endothelial cells in normal vessels that typically occurs in the head or lower and upper extremities, with a predilection for fingers, as a palpable soft-tissue mass [1-3]. In our case, thanks to HRUS we were able to exclude a flexor tendon cyst and a giant cell tumor of the tendon because the lesion was cleaved from the flexor tendon and was vascularized (differently than a tendon cyst). The definitive diagnosis is histopathological and IPEH should be distinguished from angiosarcoma, pyogenic granuloma, glomeruloid haemangioma, and Dabska tumour.

Calcifying aponeurotic fibroma is a rare, benign soft tissue neoplasm, that is generally found in the palm of the hand and in the sole of the foot $[4,5]$. This tumor is local- 
ly aggressive with the tendency to infiltrate the surrounding soft tissue and the predilection for local recurrence after the surgical excision, with a rate of recurrence of $50 \%[4,5]$. HRUS is useful for highlighting the presence of calcified tissue and to study the cleavage from the surrounding tissues (tendons, bone). It should be included in the differential diagnoses of any mass with calcification in the palm of the hand like fibromatoses, plantarpalmar fibromatosis, nodular fasciitis but also parosteal/ soft tissue chondroma, synovial sarcoma, and calcified epidermoid.

In conclusion, this case underlines the importance of HRUS and recalls the existence of these two rare lesions that should be included in the differential diagnosis.

\section{References}

1. Erol O, Ozcakar L, Uygur F, Kecik A, Ozkaya O. Intravascular papillary endothelial hyperplasia in the finger: Not a premier diagnosis. J Cutan Pathol 200;34:806-807.

2. Katzman B, Caligiuri DA, Klein DM, Nicastri AD, Chen P. Recurrent intravascular papillary endothelial hyperplasia. J Hand Surgery Br 1997;22:113-15.

3. Lee SH, Suh JS, Lim BI, Yang WI, Shin KH. Intravascular papillary endothelial hyperplasia of extremities: MR imaging findings with pathologic correlation. Eur Radiol 2004;14:822

4. Kim OH, Kim YM. Calcifying aponeurotic fibroma: case report with radiographic and MR features. Korean J Radiol 2014;15:134-139

5. Goldman RL. The cartilage analogue of fibromatosis (aponeurotic fibroma). Further observations based on 7 new cases. Cancer 1970;26:1325-1331.

\title{
A novel approach for ultrasound guided axillary nerve block: the inferior axilla technique
}

\author{
Ke-Vin Chang ${ }^{1}$, Chih-Peng Lin², Chia-Shiang Lin ${ }^{3}$, Wei-Ting Wư ${ }^{1}$, Levent Özçakar ${ }^{4}$
}

${ }^{1}$ Department of Physical Medicine and Rehabilitation and Community and Geriatric Research Center, National Taiwan University Hospital, Bei-Hu Branch and National Taiwan University College of Medicine, Taipei, Taiwan, ${ }^{2}$ Department of Anesthesiology, National Taiwan University Hospital, Taipei, Taiwan and National Taiwan University College of Medicine, Taipei, Taiwan, ${ }^{3}$ Department of Anesthesiology, Mackay Memorial Hospital, Mackay Medicine, Nursing and Management College, and Mackay Medical College, Taipei, Taiwan, ${ }^{4}$ Department of Physical and Rehabilitation Medicine, Hacettepe University Medical School, Ankara, Turkey

\section{To the Editor}

The axillary nerve originating from the posterior cord of the brachial plexus, provides sensation to the inferior glenohumeral joint, and innervates teres minor and deltoid muscles $[1,2]$. The nerve is generally entrapped due to repeated overhead activities, humeral head fracture and improper use of the axillary crutch.

Received 29.08.2017 Accepted 29.09.2017

Med Ultrason

2017, Vol. 19, No 4, 458-459, DOI: $10.11152 / \mathrm{mu}-1232$

Corresponding author: Ke-Vin Chang, MD, PhD

Department of Physical Medicine and Rehabilitation

National Taiwan University Hospital, Bei-Hu

Branch and National Taiwan University

College of Medicine, Taipei, Taiwan

E-mail: kvchang011@gmail.com
Ultrasound guided injection of the axillary nerve through the posterior shoulder has been widely used [3]. The target is the quadrilateral space bordered by the teres minor (cranially) and teres major (caudally) muscles, long head of the triceps (medially) and humeral shaft (laterally) [4]. However, the axillary nerve might also be entrapped more proximally, before its emergence from the quadrilateral space. Very few papers mention the detailed sonoanatomy of the axillary region, making the proximal intervention on the nerve challenging. The present report aims to introduce a novel approach to visualize the axillary nerve and intervene it in the inferior axilla.

First, the subject is positioned comfortably with full shoulder abduction, exposing the whole axilla (fig 1a). The linear transducer is placed horizontally on the axillary fold. The latissimus dorsi muscle is seen superfi- 


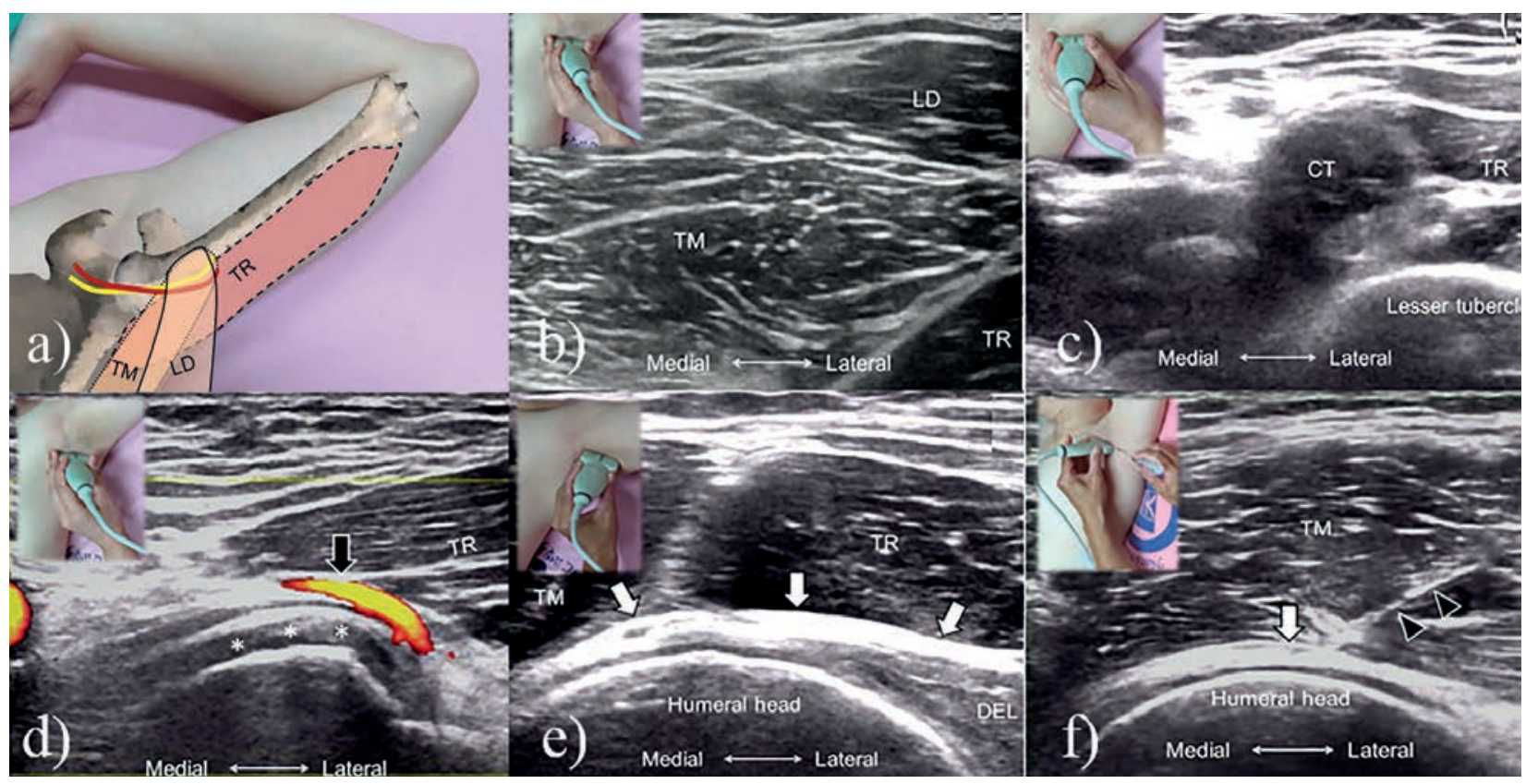

Fig 2. Illustration of the neuromuscular anatomy in the inferior axilla (a); sonoanatomy of the muscles at the level of the distal axillary fold (b) and at the lesser tubercle of the humerus (c); power Doppler imaging of the posterior circumflex artery around the humeral head (d); the long axis view of the axillary nerve in the inferior axilla (e); ultrasound guided axillary nerve block using the inferior axilla technique (f). TM, teres major; LD, latissimus dorsi; TR, triceps; CT, conjoint tendon; black arrow - posterior circumflex humeral artery; asterisk - inferior glenohumeral recess; white arrow - axillary nerve; black arrow heads - needle.

cially with the teres major muscle lying underneath (fig 1b). Relocating the transducer toward the lesser tubercle of the humerus, the tendinous portion of the latissimus dorsi muscle will become apparent. Thereafter, the teres major muscle will be seen to blend with the latissimus dorsi, becoming the conjoint tendon (fig 1c). The long head of the triceps muscle emerges laterally to the latissimus dorsi and teres major muscles and its size enlarges when the transducer approaches the lesser tubercle. The transducer is then moved medially along the long axis of the humerus until the humeral head, a spherical structure with overlying hypoechoic cartilage, is visualized. The posterior humeral circumflex artery is seen encircling the humeral head and can be traced back to the brachial artery (fig 1d). Paralleling the transducer to the posterior circumflex artery and slightly swaying it, the axillary nerve can be found coursing around the inferior glenohumeral joint capsule. The transducer can be relocated more medially to see the axillary nerve emerging from the posterior cord of the brachial plexus. Moving the transducer more laterally, the axillary nerve should be found leaving the overlying teres major muscle and coursing between the long head of triceps and deltoid muscles (fig 1e). Of note, abduction of the shoulder to nearly $180^{\circ}$ aligns the teres minor muscle along the lateral border of the scapula and to the long axis of the humerus. Importantly, the patient can be positioned side-lying with a pillow to support the posterior aspect of the injected shoulder. The needle is introduced in-plane laterally to medially and then the inferior axillary segment of the axillary nerve can be fully hydrodissected along its long axis (fig 1f, Video 1, on the journal site).

Acknowledgments: the present study is supported by National Taiwan University Hospital, Bei-Hu Branch Ministry of Science and Technology (MOST 106-2314-B-002180-MY3) and Taiwan Society of Ultrasound in Medicine.

\section{Reference}

1. Wu CH, Chang KV, Ozcakar L, et al. Sonographic tracking of the upper limb peripheral nerves: A pictorial essay and video demonstration. Am J Phys Med Rehabil 2015;94:740-747.

2. Chang KV, Lin CP, Lin CS, Wu WT, Karmakar MK, Ozcakar L. Sonographic tracking of trunk nerves: Essential for ultrasound-guided pain management and research. J Pain Res 2017;10:79-88.

3. Rothe C, Asghar S, Andersen HL, Christensen JK, Lange $\mathrm{KH}$. Ultrasound-guided block of the axillary nerve: A volunteer study of a new method. Acta Anaesthesiol Scand 2011;55:565-570.

4. Hung CY, Chang KV, Chen PT, et al. Sonoelastography for the evaluation of an axillary schwannoma in a case of quadrilateral space syndrome. Clin Imaging 2014;38:360-363. 


\title{
Non-fresh formaldehyde-embalmed cadaver: not an eligible model for ultrasound guided sacroiliac joint injection studies
}

\author{
Kamal Mezian', Ondřej Naňka ${ }^{2}$
}

${ }^{1}$ Institute of Biophysics and Informatics, ${ }^{2}$ Institute of Anatomy, Charles University in Prague, 1st Faculty Of Medicine, Prague, Czech Republic

To the Editor,

We recently conducted an ultrasound-guided sacroiliac (SI) joint intra-articular injection feasibility study. We used six non-fresh formaldehyde-embalmed wholebody human cadavers (12 SI joints). The cadavers were embalmed with a standard formaldehyde-embalming method for anatomy dissection lectures (fig 1). All injections were performed by a board-certified physical medicine and rehabilitation physician who is familiar with this method.

A portable ultrasound machine with $2-5 \mathrm{MHz}$ curvilinear array probe was used (UGEO HM70A, Samsung, Seoul, South Korea). For injection, we followed the protocol described by Chang et al [1]. However, we faced few serious difficulties. Therefore, we feel obligated to share our experience regarding our study. First, very lowquality ultrasound images were obtained. Although for SI joint injection bony landmarks are especially crucial, probe position was not always certain because of the unclear image. We also failed to improve the quality of images by injecting water on multiple tissue levels preceding the ultrasound examination. The likely explanation is the strong alteration of the tissue properties due to the embalming process. Another difficulty we experienced was the high tissue resistance on introducing the needle, having a tendency to bend and to get lost from the image.

Received 29.09.2017 Accepted 07.10.2017

Med Ultrason

2017, Vol. 19, No 4, 460-461, DOI: 10.11152/mu-1269

Corresponding author: Kamal Mezian, M.D.

$1^{\text {st }}$ Faculty of Medicine of Charles University in Prague

Institute of Biophysics and Informatics

Salmovska 1, Prague 12000

Czech Republic

Phone: +420416735234

E-mail: kamal.mezian@gmail.com

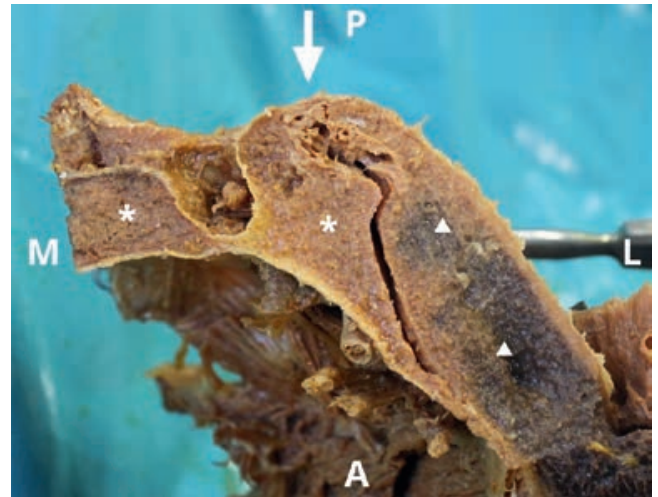

Fig 1. Cadaveric section of the sacroiliac joint. Arrow - dorsal sacroiliac ligaments; asterisks - sacrum; triangles - ilium; $\mathrm{A}$ - anterior; $\mathrm{P}$ - posterior; $\mathrm{M}$ - medial; $\mathrm{L}$ - lateral.

Likewise, the latex dye injection delivery was more difficult (high resistance) compared to the resistance that we previously found in our patients. When we dissected the cadavers one week later, we failed to identify the latex dye in four SI joints. The cause was most likely intraosseous application caused by the softening of the bone surface during the embalming process. In seven joints we found the dye peri-articularly and in one case, intraarticularly.

On the other hand, Tsui et al found the standard protocol for embalmed cadavers to be viable for ultrasound needle guidance for nerve blocks of the trunk and epidural space [2]. In a recent study Perry et al found ultrasound-guided frozen-cadavers suitable models for sacroiliac joint injection accuracy study [3]. Benkhadra et al found suitable for ultrasound-guided interventional studies [4] the embalming process according to Thiel's method (mixture consisting of monopropylene glycol, ammonium nitrate, potassium nitrate, sodium sulphite, 
boric acid, chlorocresol, and a small amount of formaldehyde).

In conclusion, we suggest to not use the non-fresh formaldehyde-embalmed cadavers for US-guided interventional studies.

\section{References}

1. Chang W-H, Lew HL, Chen CPC. Ultrasound-guided sacroiliac joint injection technique. Am J Phys Med Rehabil Assoc Acad Physiatr 2013;92:278-279.
2. Tsui B, Dillane D, Pillay J, Walji A. Ultrasound imaging in cadavers: training in imaging for regional blockade at the trunk. Can J Anaesth J Can Anesth 2008;55:105-111.

3. Perry JM, Colberg RE, Dault SL, Beason DP, Tresgallo III RA, A Cadaveric Study Assessing the Accuracy of Ultrasound Guided Sacroiliac Joint Injections, PM\&R 2016;8:1168-1172.

4. Benkhadra M, Faust A, Ladoire S, et al. Comparison of fresh and Thiel's embalmed cadavers according to the suitability for ultrasound-guided regional anesthesia of the cervical region. Surg Radiol Anat SRA 2009;31:531535. 